\title{
On the Structure of Classical Mechanics
}

\author{
Thomas William Barrett*†
}

February 21, 2013

\begin{abstract}
Jill North (North, 2009) has recently argued that Hamiltonian mechanics ascribes less structure to the world than Lagrangian mechanics does. I will argue that North's argument is not sound. In doing so, I will present some obstacles that must be navigated by anyone interested in comparing the amounts of structure that different physical theories ascribe to the world.
\end{abstract}

\section{Introduction}

Different spacetime theories ascribe different amounts of structure to spacetime. Aristotelian spacetime singles out a preferred spatial location as the center of the universe. Absolute Newtonian spacetime does not single out a preferred spatial location, but it does single out a preferred inertial frame as the rest frame. Neo-Newtonian (or Galilean) spacetime does not single out a preferred inertial frame, but it does single out a set of inertial frames as the non-accelerating frames. The list could go on. There is an intuitive sense in which each of the theories in the list ascribes less structure to spacetime than do its predecessors. ${ }^{1}$

\footnotetext{
*thomaswb@princeton.edu

${ }^{\dagger}$ I am particularly grateful to Hans Halvorson for comments on multiple drafts, for many clear discussions, and for giving me many of the basic ideas in Section 3. Thanks also to John Manchak and Jeff Barrett for comments on an earlier draft of this paper, and to Noel Swanson for helpful discussions about classical mechanics.

${ }^{1}$ See (Earman, 1989, Ch. 2).
} 
Philosophers and physicists have devoted much attention to the amounts and kinds of structure that spacetime theories ascribe to the world, but somewhat less to the amounts and kinds of structure that other physical theories ascribe to the world. In a recent paper, however, Jill North (North, 2009) undertakes the ambitious task of comparing the structure of the Hamiltonian and Lagrangian formulations of classical mechanics. Among other things, ${ }^{2}$ North argues for the following conclusion:

(LS) Hamiltonian mechanics ascribes less structure to the world than Lagrangian mechanics does.

There are, of course, general philosophical issues which turn on such questions of comparative structure. Identifying the structure that physical theories ascribe to the world could play an important role in addressing questions of theoretical equivalence. If two theories ascribe different structures to the world - if, for example, one theory singles out a spatial location as the center of the universe while another does not - then these two theories could not be equivalent reformulations of one another. They tell us different things about the structure of the world. Structural comparisons can also play an important role in parsimony arguments. All other things being equal, Ockhamist principles urge us to prefer the theory which is more structurally parsimonious.

If (LS) were true, therefore, one might use it to argue that Hamiltonian and Lagrangian mechanics are not theoretically equivalent and, furthermore, that we should prefer the Hamiltonian formulation over the Lagrangian formulation. ${ }^{3}$ Or one might use (LS) to argue for something even stronger: Hamiltonian mechanics provides us with a better description of the fundamental and objective features of the world than Lagrangian mechanics does. But of course, one must demonstrate that (LS) is actually true before drawing any of these philosophical conclusions. North's argument for (LS) runs as follows:

\footnotetext{
${ }^{2}(\mathrm{LS})$ appears as a premise in an argument that the two theories are not equivalent, and in an argument that Hamiltonian mechanics is more fundamental than Lagrangian mechanics (North, 2009, p. 76).

${ }^{3}$ As remarked in the previous footnote, North uses (LS) to argue for such conclusions.
} 
(P1) Lagrangian mechanics ascribes metric structure to the world.

(P2) Hamiltonian mechanics ascribes symplectic structure to the world.

(P3) Symplectic structure is less structure than metric structure.

$\therefore(\mathbf{L S})$

The primary goal of this paper will be to show that this argument for (LS) is not sound. I will argue that North's arguments fail to demonstrate that $(\mathrm{P} 1)$ and $(\mathrm{P} 2)$ are true. Then I will argue that even if one were to accept that (P1) and (P2) were true, (LS) would still not follow since it is not at all clear that (P3) is true. The reader might be inclined to take these remarks as cautionary: Much care must be taken in identifying and comparing the amounts of structure that different physical theories impute on the world. Some cases are harder than others. And as we will see, the case of Lagrangian and Hamiltonian mechanics is one of the hard ones.

\section{Hamiltonian mechanics imputes less structure than Lagrangian mechanics}

The first order of business is to recapitulate North's argument in support of (LS). I will first present a couple of arguments for (P1), then an argument for (P2), and then finally conclude with North's argument for (P3).

\subsection{Lagrangian mechanics is Riemannian}

Lagrangian mechanics and Hamiltonian mechanics are geometric formulations of classical mechanics. They are formulated in the language of differential geometry. The Lagrangian approach focuses on position and velocity, while the Hamiltonian approach focuses on position and momentum.

Consider a system of $n$ particles. The positions of these particles can be represented by a point $q$ in a $3 n$-dimensional differentiable manifold $\mathcal{Q}$, called configuration space. Lagrangian mechanics has as its statespace the $6 n$-dimensional tangent bundle of $\mathcal{Q}$, which we denote by $T_{*} \mathcal{Q}$. A point 
$(q, v) \in T_{*} \mathcal{Q}$ encodes the positions and velocities of all $n$ particles in the system.

In order to determine how the system evolves over time, one must specify a Lagrangian. The Lagrangian of a system is a smooth function $L: T_{*} \mathcal{Q} \rightarrow \mathbb{R}$ which encodes some of the system's energy properties. ${ }^{4}$ In most applications of Lagrangian mechanics, the Lagrangian of a system is defined as the system's kinetic energy minus its potential energy. The intuition behind it is something like the following:

Kinetic energy measures how much is 'happening' — how much our system is moving around. Potential energy measures how much could happen, but isn't yet [...]. So, the Lagrangian measures something we could vaguely refer to as the 'activity' or 'liveliness' of a system: the higher the kinetic energy the more lively the system, the higher the potential energy the less lively. (Baez, 2005, p. 7)

This intuitive interpretation of the Lagrangian helps to motivate the dynamics of the theory: Systems like to evolve along paths in $T_{*} \mathcal{Q}$ which minimize the integral of the Lagrangian — they like to minimize their total 'activity' or 'liveliness'. 5

One might at first try to make the following naive argument that Lagrangian mechanics ascribes Riemannian metric structure to the world.

Argument 1 (Naive Argument). In applications of Lagrangian mechanics we need to define things like lengths of curves or angles in $\mathcal{Q}$. If we do not have a metric $g_{q}$ on $\mathcal{Q}$, then we cannot define such things. Therefore, Lagrangian mechanics requires a metric $g_{q}$ on its configuration space $\mathcal{Q}$.

\footnotetext{
${ }^{4}$ And the system then evolves according to the Euler-Lagrange equations

$$
\frac{d}{d t} \frac{\partial L}{\partial \dot{q}^{i}}=\frac{\partial L}{\partial q^{i}}
$$

where $q^{i}$ and $\dot{q}^{i}$ are position and velocity coordinates, respectively, on $T_{*} \mathcal{Q}$. It turns out that the dynamics of Lagrangian mechanics - the way that the theory states that systems will evolve - is not important in North's argument.

${ }^{5}$ And this behavior is guaranteed by the Euler-Lagrange equations in the previous footnote.
} 
North, however, does not have such an argument in mind. This argument clearly does not serve her purposes. Remember that we are trying to argue for (LS); we are trying to argue that Hamiltonian mechanics ascribes less structure to the world than does Lagrangian mechanics. Argument 1 does not help us to argue for (LS). In applications of Hamiltonian mechanics one will also need to define things like lengths of curves and angles in $\mathcal{Q}$. Argument 1 will therefore imply that Hamiltonian mechanics requires a metric on its configuration space $\mathcal{Q}$ also. And this is more than we want to argue for. If both Hamiltonian and Lagrangian mechanics posit metric structure, then it will be hard to argue that Hamiltonian mechanics posits less structure than Lagrangian mechanics does. Argument 1 will not do. North's argument is more like the following:

Argument 2 (North's Argument). In most applications of Lagrangian mechanics, the Lagrangian of a system is defined as the system's kinetic energy minus its potential energy. In fact, in many cases the Lagrangian is defined by

$$
L(q, v)=\frac{1}{2} g_{q}(v, v)-V(q),
$$

where $V: \mathcal{Q} \rightarrow \mathbb{R}$ is a smooth potential. And this definition presupposes a metric $g_{q}: T_{q} \mathcal{Q} \times T_{q} \mathcal{Q} \rightarrow \mathbb{R}$ on the configuration space $\mathcal{Q}$. Therefore, Lagrangian mechanics requires a metric $g_{q}$ on its configuration space $\mathcal{Q}$.

It is in this sense, then, that Lagrangian mechanics ascribes Riemannian metric structure to the world. In specifying a Lagrangian for a system, one often requires that configuration space $\mathcal{Q}$ has metric structure. Having presented North's argument for (P1), we now turn to (P2).

\subsection{Hamiltonian mechanics is Symplectic}

Consider again an $n$ particle system. Hamiltonian mechanics has as its statespace the $6 n$-dimensional cotangent bundle of configuration space $\mathcal{Q}$, denoted by $T^{*} \mathcal{Q}$. A point $(q, \omega) \in T^{*} \mathcal{Q}$ encodes the positions and momenta of all $n$ particles in the system. ${ }^{6}$ As in the Lagrangian approach, we now

\footnotetext{
${ }^{6}$ One might wonder why momentum is represented by a covector rather than a vector. After all, velocity is most naturally thought of as a vector, and momentum is simply the
} 
need to specify some energy conditions to be able to say how systems evolve over time. We do this by specifying a smooth function $H: T^{*} \mathcal{Q} \rightarrow \mathbb{R}$, called the Hamiltonian, which encodes the total energy of the system in question. ${ }^{7}$

The argument for (P2) is simple: The cotangent bundle $T^{*} \mathcal{Q}$ actually has a natural symplectic structure associated with it. This symplectic structure is constructed by first noticing the following fact.

Fact 1. There is a canonical covector field $\theta_{(q, \omega)}$ on $T^{*} \mathcal{Q}$, called the Liouville (or Poincaré, or tautological) 1-form.

The construction of the Liouville 1-form is straightforward. The cotangent bundle $T^{*} \mathcal{Q}$ comes equipped with a smooth projection $\pi: T^{*} \mathcal{Q} \rightarrow \mathcal{Q}$ defined by

$$
\pi(q, \omega)=q .
$$

Given a point $(q, \omega) \in T^{*} \mathcal{Q}$, the projection $\pi$ induces a linear pullback $\pi^{*}: T_{q}^{*} \mathcal{Q} \rightarrow T_{(q, \omega)}^{*} T^{*} \mathcal{Q}$. Now to each point $(q, \omega) \in T^{*} \mathcal{Q}$, we want to assign a covector — that is, an element of $T_{(q, \omega)}^{*} T^{*} \mathcal{Q}$. So given such a point $(q, \omega) \in T^{*} \mathcal{Q}$ we define

$$
\theta_{(q, \omega)}=\pi^{*}(\omega)
$$

The covector field $\theta_{(q, \omega)}$ on $T^{*} \mathcal{Q}$ is the Liouville 1-form.

Of course, we do not yet have a symplectic form on $T^{*} \mathcal{Q}$; we only have a covector field. But it is not hard to get a symplectic form out of $\theta_{(q, \omega)}$. We simply take its exterior derivative. We define

$$
\Omega=d \theta_{(q, \omega)}
$$

And as one can easily verify, $\Omega$ is a symplectic form on $T^{*} \mathcal{Q}$. $\Omega$ is the canonical symplectic form on the cotangent bundle.

product of mass - a scalar - and velocity. Momentum is viewed as a covector, however, because of how it is defined in Lagrangian mechanics: $p:=\frac{\partial L}{\partial \dot{q}}$, which most naturally lives in the cotangent space. See (Tao, 2008) for a nice discussion.

${ }^{7}$ The dynamics of Hamiltonian mechanics are given by Hamilton's equations of motion:

$$
\dot{q}^{i}=\frac{\partial H}{\partial p_{i}} ; \quad \dot{p}^{i}=-\frac{\partial H}{\partial q_{i}} .
$$

See (Baez, 2005, Ch. 4). 
It is in precisely this sense, therefore, that Hamiltonian mechanics ascribes symplectic structure to the world. The statespace of Hamiltonian mechanics has a natural symplectic structure. This structure essentially 'comes for free' since there is a natural symplectic form $\Omega$ associated with the cotangent bundle of any manifold.

\subsection{Riemannian $>$ Symplectic}

We have argued that Lagrangian mechanics imputes Riemannian metric structure, while Hamiltonian mechanics imputes symplectic structure. Now all that remains in this recapitulation of North's argument is to argue for (P3), that Riemannian metric structure is, in some sense, more structure than symplectic structure.

It is clear what North's argument is for (P3). A symplectic form $\Omega$ on a $2 n$-dimensional manifold determines a volume form $\Omega^{n}=\Omega \wedge \ldots \wedge \Omega$ on the manifold, the $n$-fold wedge product of $\Omega$ with itself. So the statespace of Hamiltonian mechanics has volume structure, while the statespace of Lagrangian mechanics has metric structure. North reminds us that while distance determines volume, ${ }^{8}$ volume does not determine distance.

[...] there is a clear sense in which a space with a metric structure has more structure than one with just a volume element. Metric structure comes with, or determines, or presupposes, a volume structure, but not the other way around. [... Intuitively, knowing the distances between the points in a space will give you the volumes of the regions, but the volumes will not determine the distances. Metric structure adds a further level of structure. (North, 2009, p. 74-75)

Since the statespace of Lagrangian mechanics has full-blown metric structure, while the statespace of Hamiltonian mechanics only has volume structure, North concludes that Hamiltonian mechanics imputes less structure

\footnotetext{
${ }^{8} \mathrm{~A}$ metric does not determine a volume form because a volume form comes with an orientation and a metric does not give you that. For example, one can put a metric on the Möbius strip, but one cannot put a volume form on it. A metric, however, does determine something very close to a volume form, a volume element (which is equal to the absolute value of a volume form). A volume element can be put on any manifold, orientable or not.
} 
on the world than does Lagrangian mechanics. This completes North's argument for (LS).

\section{Hamiltonian mechanics does not impute less struc- ture than Lagrangian mechanics}

The goal of this section will be to show that North's argument for (LS), as presented in the previous section, is not sound. I will first argue that, in general, Lagrangian mechanics does not posit metric structure. Then I will show that whenever the Lagrangian statespace has metric structure, the Hamiltonian statespace has more structure than mere symplectic structure. Finally, I will conclude by arguing that there is a compelling sense in which metric structure is not more structure than symplectic structure.

\subsection{Lagrangian mechanics has less than metric structure}

The Lagrangian statespace does not always have metric structure. In general, specifying a Lagrangian for a system neither presupposes nor gives rise to a metric on configuration space. But before arguing for this, let me assuage another potential worry that one might have with North's argument for (LS).

\subsubsection{A Potential Worry}

One might have noticed an asymmetry in the above arguments. The argument for (P1) concludes that specifying a Lagrangian requires a metric on the configuration space $\mathcal{Q}$. The argument for (P2) concludes that there is a natural symplectic form on the cotangent bundle $T^{*} \mathcal{Q}$. We are therefore comparing metric structure on $\mathcal{Q}$ to symplectic structure on $T^{*} \mathcal{Q}$. This might strike one as problematic. But this worry can be assuaged, at least in part, by recognizing the following fact.

Fact 2. A metric $g_{q}$ on $\mathcal{Q}$ can be 'pulled back' onto $T_{*} \mathcal{Q}$ using the smooth projection $\pi$. 
Suppose that, as North argues, Lagrangian mechanics requires a metric $g_{q}$ on $\mathcal{Q}$. The projection $\pi: T_{*} \mathcal{Q} \rightarrow \mathcal{Q}$ induces a linear pushforward from the tangent spaces of $T_{*} \mathcal{Q}$ to the tangent spaces of $\mathcal{Q}$ denoted by $\pi_{*}: T_{(q, v)} T_{*} \mathcal{Q} \rightarrow T_{q} \mathcal{Q}$, for each $(q, v) \in T_{*} \mathcal{Q}$. The metric $g_{q}$ on $\mathcal{Q}$ and the map $\pi_{*}$ naturally determine a metric $g_{(q, v)}^{\prime}$ on $T_{*} \mathcal{Q}$ defined by

$$
g_{(q, v)}^{\prime}(x, y)=g_{q}\left(\pi_{*}(x), \pi_{*}(y)\right)
$$

where $x, y \in T_{(q, v)} T_{*} \mathcal{Q}$ for some $(q, v) \in T_{*} \mathcal{Q}$.

This gives us at least a semblance of symmetry in the two arguments. In Lagrangian mechanics, we have metric structure on $T_{*} \mathcal{Q},{ }^{9}$ while in Hamiltonian mechanics we have symplectic structure on $T^{*} \mathcal{Q}$. This asymmetry between the argument on the Lagrangian side and the argument on the Hamiltonian side does not, therefore, impinge on North's conclusion.

\subsubsection{General Lagrangians}

What does impinge on North's conclusion, however, is that in general the Lagrangian statespace does not actually have metric structure. In general, (P1) is not true. As North rightly points out (North, 2009, p. 73), in the completely general case the Lagrangian does not make reference to a metric on $\mathcal{Q}$. The Lagrangian is merely an arbitrary smooth scalar function $L$ : $T_{*} \mathcal{Q} \rightarrow \mathbb{R}$. It need not be of the form

$$
L(q, v)=\frac{1}{2} g_{q}(v, v)-V(q),
$$

with $g_{q}$ a metric on $\mathcal{Q}$ and $V: \mathcal{Q} \rightarrow \mathbb{R}$ a smooth potential. In general, specifying a Lagrangian $L$ for a system simply does not require a metric $g_{q}$ on configuration space.

One might try to argue, however, that although specifying a Lagrangian $L: T_{*} \mathcal{Q} \rightarrow \mathbb{R}$ neither presupposes nor requires a metric, it in some sense gives rise to or determines or implicitly defines a metric. A potential argument for (P1) along these lines is suggested by the following two facts.

\footnotetext{
${ }^{9}$ And, of course, we still have metric structure on $\mathcal{Q}$.
} 
Fact 3. Given a point $q \in \mathcal{Q}$, a smooth function $L: T_{*} \mathcal{Q} \rightarrow \mathbb{R}$ gives rise to a $\operatorname{map} \mathcal{L}_{L}^{q}: T_{q} \mathcal{Q} \rightarrow T_{q}^{*} \mathcal{Q}$ defined by:

$$
\mathcal{L}_{L}^{q}(v) \cdot w=\left.\frac{d}{d t} L(q, v+t w)\right|_{t=0},
$$

for each $v, w \in T_{q} \mathcal{Q}$, where - denotes the application of a covector to a vector. The map $\mathcal{L}_{L}: T_{*} \mathcal{Q} \rightarrow T^{*} \mathcal{Q}$ defined by $(q, v) \mapsto\left(q, \mathcal{L}_{L}^{q}(v)\right)$ is usually called the Legendre transformation associated with $L .^{10}$

The Legendre transformation allows one to 'translate' between the Lagrangian framework and the Hamiltonian framework. Intuitively, the covector $\mathcal{L}_{L}^{q}(v)$ encodes the momenta of the particles in a system given that they have velocity $v \in T_{q} \mathcal{Q}$ and the system has Lagrangian $L$.

As following fact shows, certain maps between a vector space and its dual naturally give rise to additional structure on the vector space.

Fact 4. Let $V$ be a finite dimensional real vector space, and $V^{*}$ its dual. Let $f: V \rightarrow V^{*}$ be a vector space isomorphism. Then $f$ gives rise to a nondegenerate bilinear form $g: V \times V \rightarrow \mathbb{R}$ on $V$ defined by

$$
g(x, y)=f(x) \cdot y
$$

where $\cdot$ again denotes the application of a covector to a vector. ${ }^{11}$

Fact 4 is illustrated in the following example. This example demonstrates that in some cases, an isomorphism between a vector space and its dual actually induces an inner product on the vector space.

Example 1. Let $V$ be an $n$-dimensional real vector space, and $V^{*}$ its dual.

\footnotetext{
${ }^{10}$ For a nice geometrical discussion of the Legendre transformation see (Mac Lane, 1970). We will restrict our attention here to 'nice' Lagrangians - ones whose Legendre transformations $\mathcal{L}_{L}$ are actually diffeomorphisms between the tangent and cotangent bundles. Such Lagrangians are typically called hyperregular (Abraham and Marsden, 1978, §3.6).

${ }^{11}$ Although the notation $g$ is suggestive, one should note that for an arbitrary isomorphism $f$ the associated bilinear form $g$ need not be an inner product on $V$.
} 
Let $\left\{e_{i}\right\}$ be a basis for $V$. For each $i \in\{1, \ldots, n\}$ let $e^{i} \in V^{*}$ be defined by

$$
e^{i} \cdot e_{j}=\left\{\begin{array}{rr}
1 & \text { if } i=j \\
0 & \text { otherwise }
\end{array} .\right.
$$

Now define the isomorphism $f$ by $f\left(e_{i}\right)=e^{i}$ and extending linearly. By Fact $4, f$ induces a nondegenerate bilinear form $g$ on $V$. As one can easily verify, in this case $g$ is the inner product which makes the basis $\left\{e_{i}\right\}$ orthonormal.

Fact 3 and Fact 4 together suggest a potential argument for $(\mathrm{P} 1)$. If at each point $q \in \mathcal{Q}$, the map $\mathcal{L}_{L}^{q}: T_{q} \mathcal{Q} \rightarrow T_{q}^{*} \mathcal{Q}$ is a vector space isomorphism, then by Fact $4, \mathcal{L}_{L}^{q}$ would naturally give rise to a nondegenerate bilinear form on $T_{q} \mathcal{Q}$. And if for each $q \in \mathcal{Q}, \mathcal{L}_{L}^{q}$ is like the isomorphism $f$ in Example $1,{ }^{12}$ then $L$ would naturally induce an inner product on each of the tangent spaces of $\mathcal{Q}$ - that is, a metric on $\mathcal{Q}$. It would be in this sense, then, that we could say that specifying a Lagrangian $L$ gave rise to a metric on $\mathcal{Q}$.

Unfortunately, however, this argument for (P1) does not work. As the following example illustrates, the map $\mathcal{L}_{L}^{q}$ is not always a vector space isomorphism.

Example 2. Let $\mathcal{Q}=\mathbb{R}$. Define the Lagrangian $L: T_{*} \mathbb{R} \rightarrow \mathbb{R}$ by

$$
L(x, v)=v^{4}
$$

for each $(x, v) \in T_{*} \mathbb{R}$. Given $x \in \mathbb{R}, \mathcal{L}_{L}^{x}: T_{x} \mathbb{R} \rightarrow T_{x}^{*} \mathbb{R}$ is given by

$$
\mathcal{L}_{L}^{x}(v) \cdot w=\left.\frac{d}{d t} L(x, v+t w)\right|_{t=0}=\left.\frac{d}{d t}(v+t w)^{4}\right|_{t=0}=\left(4 v^{3}\right)(w)
$$

for each $v, w \in T_{x} \mathbb{R}$. So we conclude that $\mathcal{L}_{L}^{x}(v)=4 v^{3}$. The map $\mathcal{L}_{L}$ is a diffeomorphism, but for every $x \in \mathbb{R}, \mathcal{L}_{L}^{x}$ is not linear and thus not a vector space isomorphism.

This attempt to rescue premise (P1) therefore does not work. The maps $\mathcal{L}_{L}^{q}: T_{q} \mathcal{Q} \rightarrow T_{q}^{*} \mathcal{Q}$ induced by the Lagrangian $L$ are not always vector space

\footnotetext{
${ }^{12}$ By which I mean, if the nondegenerate bilinear form induced by $\mathcal{L}_{L}^{q}$ were symmetric like $g$ in Example 1 .
} 
isomorphisms. Therefore, one cannot use Fact 4 to conclude that a Lagrangian gives rise to nondegenerate bilinear forms on the tangent spaces of $\mathcal{Q}$. In general, specifying a Lagrangian does not presuppose a metric on configuration space. And Example 2 shows that specifying a Lagrangian also does not give rise to a metric on $\mathcal{Q}$, at least in the sense described above. It is thus exceedingly hard to see any sense in which (P1) is true.

\subsection{Hamiltonian mechanics (often) has more than symplectic structure}

One might respond to these concerns about (P1), however, simply by stipulating that the scope of Lagrangian mechanics should be restricted to only include systems with Lagrangians which do presuppose a metric on $\mathcal{Q}$. One might argue, for example, that systems with strange Lagrangians - like the one in Example 2 - are not physically reasonable systems. After all, many common systems that one deals with in Lagrangian mechanics do have Lagrangians of the form $L(q, v)=\frac{1}{2} g_{q}(v, v)-V(q)$. One might argue that it is in this sense, therefore, that (P1) is true: Most of the physically reasonable systems dealt with in Lagrangian mechanics have Lagrangians which presuppose a metric on configuration space.

In this section, however, I will argue that even if one restricts the scope of Lagrangian mechanics to deal only with systems which presuppose a metric on $\mathcal{Q}$, North's argument for (LS) still does not go through. There is a strong sense in which whenever Lagrangian mechanics posits metric structure, Hamiltonian mechanics does the same. I will first argue that whenever there is a metric on the Lagrangian side of things, the metric naturally induces a dual structure on the Hamiltonian side of things. Then I will show that many simple Hamiltonians presuppose this dual structure in exactly the same way that many simple Lagrangians presuppose a metric.

\subsubsection{A Dual Structure on the Hamiltonian Side}

Suppose that, as North has argued, in Lagrangian mechanics the configuration space $\mathcal{Q}$ has a metric $g_{q}$. The following fact is then crucial in comparing the structures of the Lagrangian and Hamiltonian statespaces. 
Fact 5. Given a point $q \in \mathcal{Q}$, the metric $g_{q}$ induces a vector space isomorphism $F_{g}^{q}: T_{q} \mathcal{Q} \rightarrow T_{q}^{*} \mathcal{Q}$, defined for each $v \in T_{q} \mathcal{Q}$ by

$$
F_{g}^{q}(v)=g_{q}(v,-)
$$

where the covector $g_{q}(v,-) \in T_{q}^{*} \mathcal{Q}$ acts on arbitrary vectors $x \in T_{q} \mathcal{Q}$ by $x \mapsto g_{q}(v, x)$. The map $F_{g}: T_{*} \mathcal{Q} \rightarrow T^{*} \mathcal{Q}$ defined by $F_{g}:(q, v) \mapsto\left(q, F_{g}^{q}(v)\right)$ is a diffeomorphism, usually called the musical isomorphism.

The isomorphism $F_{g}$ lets us essentially 'move' the metric structure $g_{q}$ from the Lagrangian side of things onto the Hamiltonian side of things. The metric $g_{q}$ allows one to determine lengths of and angles between vectors which are supposed to encode velocities - at points of $\mathcal{Q}$. And it turns out that $g_{q}$ naturally gives rise to a corresponding structure $g_{q}^{*}$ which allows one to determine lengths of and angles between covectors - which are supposed to encode momenta - at points of $\mathcal{Q}$.

The metric $g_{q}$ is a smooth assignment of inner products to the tangent spaces of $\mathcal{Q} ; g_{q}^{*}$ is a smooth assignment of inner products to the cotangent spaces of $\mathcal{Q}$. It is easy to define how $g_{q}^{*}$ acts on pairs of covectors using the fact that for each $q \in \mathcal{Q}, F_{g}^{q}$ is an isomorphism and thus invertible and linear:

$$
g_{q}^{*}(\alpha, \beta)=g_{q}\left(\left(F_{g}^{q}\right)^{-1}(\alpha),\left(F_{g}^{q}\right)^{-1}(\beta)\right),
$$

for every $\alpha, \beta \in T_{q}^{*} \mathcal{Q} .{ }^{13}$ Whenever there is a metric $g_{q}$ on the Lagrangian side of things, therefore, a dual structure $g_{q}^{*}$ naturally arises on the Hamiltonian side of things. If (P1) is true and the Lagrangian statespace has metric structure, then the Hamiltonian statespace has more structure than mere symplectic structure.

\footnotetext{
${ }^{13}$ Two remarks are worth being made here. First, as anyone familiar with geometry will have already recognized, in coordinates the components of $g_{q}$ are usually written $g_{\mathrm{ij}}$; the components of $g_{q}^{*}$ are usually written $g^{\mathrm{ij}}$. And second, it is important to note that the metrical structure $g_{q}^{*}$ is not just some arbitrary assignment of inner products to the cotangent spaces of $\mathcal{Q}$. Given a metric $g_{q}, g_{q}^{*}$ arises in a perfectly natural way. This is the sense in which I say $g_{q}$ determines, or gives rise to, $g_{q}^{*}$.
} 


\subsubsection{Simple Hamiltonians}

And there is, in fact, a second way to see this. Notice that North's argument for (LS) does not compare the structures of Lagrangian and Hamiltonian mechanics on level footing. Argument 2 demonstrates that Lagrangian mechanics has metric structure by considering both the statespace $T_{*} \mathcal{Q}$ and the Lagrangian $L: T_{*} \mathcal{Q} \rightarrow \mathbb{R}$ defined on that statespace. The argument that Hamiltonian mechanics has symplectic structure, however, only relies on the Hamiltonian statespace $T^{*} \mathcal{Q}$. It does not take into account the Hamiltonian $H: T^{*} \mathcal{Q} \rightarrow \mathbb{R}$ defined on the statespace.

This might strike one as strange. Lagrangians and Hamiltonians serve similar purposes in Lagrangian and Hamiltonian mechanics. They encode certain energy conditions of the systems in question and, in conjunction with the dynamical laws, dictate how these systems will evolve over time. If in determining the structure of the Lagrangian statespace, one considers the Lagrangian $L$, then in determining the structure of the Hamiltonian statespace one should consider the Hamiltonian $H$. When this asymmetry is remedied, one can see that insofar as Lagrangian mechanics imputes the

structure $g_{q}$, Hamiltonian mechanics imputes the structure $g_{q}^{*}$ in exactly the same way.

It will be convenient to first introduce some terminology. A simple mechanical system, defined as follows, is precisely the kind of Lagrangian system which is considered above in Argument 2 for (P1).

Definition 1 (Simple Mechanical System). A simple mechanical system is a quadruple $\left(\mathcal{Q}, g_{q}, V, L\right)$, where

- $\mathcal{Q}$ is a differentiable manifold, the configuration space for the system.

- $g_{q}: T_{q} \mathcal{Q} \times T_{q} \mathcal{Q} \rightarrow \mathbb{R}$ is a Riemannian metric on $\mathcal{Q}$.

- $V: \mathcal{Q} \rightarrow \mathbb{R}$ is a smooth potential on $\mathcal{Q}$.

- $L: T_{*} \mathcal{Q} \rightarrow \mathbb{R}$ is the Lagrangian of the system, and is defined by

$$
L(q, v)=\frac{1}{2} g_{q}(v, v)-V(q)
$$


Given a Lagrangian system, there is a way to translate it into a Hamiltonian system. ${ }^{14}$ The key is to turn the Lagrangian $L: T_{*} \mathcal{Q} \rightarrow \mathbb{R}$ into a Hamiltonian $H: T^{*} \mathcal{Q} \rightarrow \mathbb{R}$. Recall that the Hamiltonian $H$ for a system is supposed to encode the system's total energy. And of course, there is a way to define the total energy of a system in Lagrangian mechanics.

Definition 2 (Total Energy). Given a system with Lagrangian $L: T_{*} \mathcal{Q} \rightarrow$ $\mathbb{R}$, the total energy $E_{L}: T_{*} \mathcal{Q} \rightarrow \mathbb{R}$ of the system is defined by

$$
E_{L}(q, v)=\mathcal{L}_{L}^{q}(v) \cdot v-L(q, v)
$$

for each $(q, v) \in T_{*} \mathcal{Q} .^{15}$

As was remarked earlier, the Legendre transformation $\mathcal{L}_{L}: T_{*} \mathcal{Q} \rightarrow T^{*} \mathcal{Q}$ is a 'translation' between Lagrangian and Hamiltonian mechanics. The Hamiltonian $H: T^{*} \mathcal{Q} \rightarrow \mathbb{R}$ associated with a given Lagrangian $L: T_{*} \mathcal{Q} \rightarrow \mathbb{R}$ is simply defined as the translation of the total energy $E_{L}$ :

$$
H=E_{L} \circ \mathcal{L}_{L}^{-1}
$$

The Hamiltonian $H$ associated with a simple mechanical system requires or presupposes $g_{q}^{*}$ in exactly the same way that the Lagrangian $L$ associated with a simple mechanical system requires or presupposes $g_{q}$. This fact can be seen in two simple steps. First notice that for simple mechanical systems, the Legendre transformation associated with $L$ is the same as the musical isomorphism associated with $g_{q}$.

Lemma 1. Let $\left(Q, g_{q}, V, L\right)$ be a simple mechanical system. Then $\mathcal{L}_{L}=F_{g}$.

\footnotetext{
${ }^{14}$ In general, the Lagrangian $L$ of the system must be sufficiently 'nice' for this translation to work. More precisely, the Legendre transformation $\mathcal{L}_{L}$ must be a diffeomorphism. Recall that we are restricting attention to such 'nice' Lagrangians here.

${ }^{15}$ See (Abraham and Marsden, 1978, §3.5).
} 
Proof. At each point $q \in \mathcal{Q}$, the map $\mathcal{L}_{L}^{q}$ is given by

$$
\begin{aligned}
\mathcal{L}_{L}^{q}(v) \cdot w & =\left.\frac{d}{d t} L(q, v+t w)\right|_{t=0} \\
& =\left.\frac{d}{d t}\left(\frac{1}{2} g_{q}(v+t w, v+t w)-V(q)\right)\right|_{t=0} \\
& =\left.\frac{d}{d t}\left(\frac{1}{2} g_{q}(v, v)+t g_{q}(v, w)+\frac{t^{2}}{2} g_{q}(w, w)-V(q)\right)\right|_{t=0} \\
& =g_{q}(v, w)=F_{g}^{q}(v) \cdot w .
\end{aligned}
$$

Since this holds for arbitrary $v, w \in T_{q} \mathcal{Q}$, it must be that $\mathcal{L}_{L}=F_{g}$.

Then notice that the form of the Hamiltonian for a simple mechanical system is perfectly dual to the form of the system's Lagrangian.

Proposition 1. Let $\left(Q, g_{q}, V, L\right)$ be a simple mechanical system. The Hamiltonian $H: T^{*} \mathcal{Q} \rightarrow \mathbb{R}$ associated with this system is given by

$$
H(q, \omega)=\frac{1}{2} g_{q}^{*}(\omega, \omega)+V(q)
$$

where $g_{q}^{*}: T_{q}^{*} \mathcal{Q} \times T_{q}^{*} \mathcal{Q} \rightarrow \mathbb{R}$ is defined as in equation (1).

Proof. Notice that for a simple mechanical system, the energy $E_{L}: T_{*} \mathcal{Q} \rightarrow \mathbb{R}$ is given by

$$
E_{L}(q, v)=\mathcal{L}_{L}^{q}(v) \cdot v-L(q, v)=F_{g}^{q}(v) \cdot v-L(q, v)=\frac{1}{2} g_{q}(v, v)+V(q),
$$

where the second equality follows from Lemma 1, and the third from Definition 1 and Fact 5. The Hamiltonian $H$ associated with the system is therefore given by

$$
\begin{aligned}
H(q, \omega) & =E_{L} \circ \mathcal{L}_{L}^{-1}(q, \omega) \\
& =E_{L} \circ F_{g}^{-1}(q, \omega) \\
& =\frac{1}{2} g_{q}\left(\left(F_{g}^{q}\right)^{-1}(\omega),\left(F_{g}^{q}\right)^{-1}(\omega)\right)+V(q) \\
& =\frac{1}{2} g_{q}^{*}(\omega, \omega)+V(q),
\end{aligned}
$$


where the last equality follows from the definition of $g_{q}^{*}$ in equation (1).

In light of Fact 5 and Proposition 1, it is hard to see any sense in which Lagrangian mechanics could impute metric structure without Hamiltonian mechanics doing the same. If Lagrangian mechanics presupposes a metric $g_{q}$ on configuration space $\mathcal{Q}$, then Fact 5 shows that $g_{q}$ naturally induces the corresponding structure $g_{q}^{*}$ on the Hamiltonian statespace. And furthermore, Proposition 1 shows that simple Hamiltonians make reference to $g_{q}^{*}$ in precisely the same way that simple Lagrangians make reference to $g_{q}$.

Recall the two arguments for (P1) presented above. Argument 2 is, in fact, like Argument 1. Argument 1 failed because it demonstrated too much; it implied that both Lagrangian and Hamiltonian mechanics have metric structure. The same goes for Argument 2. Insofar as Argument 2 demonstrates that Lagrangian mechanics has metric structure, it demonstrates the same of Hamiltonian mechanics. If (P1) is true, then North's argument for (LS) does not go through. ${ }^{16}$ The argument is self-undermining.

\subsection{Comparing Lagrangian and Hamiltonian Structures}

Even if there were a sense in which Lagrangian mechanics had metric structure without Hamiltonian mechanics having the same, North's argument for (LS) would quickly encounter another obstacle. It is not clear that symplectic structure is, in fact, less structure than metric structure. According to one plausible criterion for comparing amounts of mathematical structure, (P3) is not true. ${ }^{17}$

\subsubsection{Counting Mathematical Structure}

North counts the structure that a physical theory ascribes to the world by looking to the models of the theory and the mathematical structure of those models. Given a mathematical object, North suggests, there is a particularly natural way to assess the amount of structure that this object has (North,

\footnotetext{
${ }^{16}$ If (P1) is true and North's argument for (P2) is sound, then Hamiltonian mechanics imputes metric structure and symplectic structure on the world. And this will clearly not serve the same purpose in North's argument as (P2) did.

${ }^{17}$ In this section I expand upon a remark made in (Swanson and Halvorson, 2012).
} 
2009, p. 87). One simply looks to the automorphisms (or symmetries) of the object. Automorphisms are structure preserving maps from a mathematical object to itself. The idea is this: If an mathematical object has more automorphisms, then it has less structure that the automorphisms need to preserve; if it has fewer automorphisms, then the object has more structure that the automorphisms need to preserve. The amount of structure that a mathematical object has is inversely proportional to, in some sense, the size of the object's automorphism group.

We therefore count the structure of mathematical objects, like the statespaces of Lagrangian and Hamiltonian mechanics, with something like the following principle in mind:

(SYM) A mathematical object $X$ has more structure than a mathematical object $Y$ if $\operatorname{Aut}(X)$ is 'smaller than' $\operatorname{Aut}(Y) \cdot{ }^{18}$

Of course, SYM is not useful until we spell out precisely what we mean by 'smaller than.' As a first attempt, North suggests that we use the dimension of the automorphism groups as the relevant measure (North, 2009, p. 87). Consider the following criterion for comparing structure:

(DIM) A mathematical object $X$ has more structure than a mathematical object $Y$ if $\operatorname{dim}(\operatorname{Aut}(X))<\operatorname{dim}(\operatorname{Aut}(Y))$.

Indeed, when we apply DIM to one particular case, it gives the intuitive answer. According to DIM, an $n$-dimensional vector space $V$ has less structure than an $n$-dimensional inner product space $(V,\langle\rangle$,$) . This is good. An$ inner product space intuitively has more structure than a mere vector space; it has inner product structure in addition to vector space structure.

But DIM suffers from a couple of shortcomings. First of all, it is not completely general. There are many mathematical objects whose automorphism group does not in any sense have a dimension. Groups do not in general have a dimension. ${ }^{19}$ Furthermore, DIM gives some puzzling answers. Consider the vector space $\mathbb{R}$ and the vector space $\mathbb{R}^{2}$. As one can easily verify, according to DIM, $\mathbb{R}^{2}$ has less structure than $\mathbb{R}$. This is a strange verdict. DIM,

\footnotetext{
${ }^{18}$ Where $\operatorname{Aut}(X)$ is the group of automorphisms of $X$.

${ }^{19}$ One might try to compare group orders, but as one can easily verify with the case of $V$ vs. $(V,\langle\rangle$,$) , this criterion will not always give the intuitive verdict.$
} 
therefore, seems unappealing as a general criterion for comparing amounts of mathematical structure.

The following example of vector space structure as compared to inner product space structure, however, suggests another more promising way to make SYM precise.

Example 3 (Vector Space vs. Inner Product Space). Let $V$ be a vector space and $g$ an inner product on $V$. As mentioned above, there is an intuitive sense in which $(V, g)$ has more structure than $V$. It has all of the vector space structure of $V$ along with additional inner product structure. And note that every automorphism of $(V, g)$ is also an automorphism of $V$, but there are some automorphisms of $V$ which are not automorphisms of $(V, g) \cdot{ }^{20}$

The automorphism group of $(V,\langle\rangle$,$) is properly contained in the auto-$ morphism group of $V$. This suggests the following revision of SYM:

(SYM*) A mathematical object $X$ has more structure than a mathematical object $Y$ if $\operatorname{Aut}(X) \subsetneq \operatorname{Aut}(Y)$.

It turns out that SYM* provides the intuitive verdict for many easy cases of structural comparison. In addition to Example 3, the following are some of the cases that $\mathrm{SYM}^{*}$ answers correctly:

- A set $X$ has less structure than a group $(X, \cdot)$.

- A set $X$ has less structure than a topological space $(X, \tau)$.

- A differentiable manifold $\left(\mathcal{M}, U_{\alpha}, \varphi_{\alpha}\right)$ has less structure than a Riemannian manifold $\left(\mathcal{M}, U_{\alpha}, \varphi_{\alpha}, g_{p}\right)$.

Furthermore, $\mathrm{SYM}^{*}$ provides the intuitive verdict when applied to the mathematical models of the classical spacetime theories mentioned at the beginning of this paper. According to $\mathrm{SYM}^{*}$, Aristotelian spacetime has more structure than absolute Newtonian spacetime, which in turn has more

\footnotetext{
${ }^{20}$ This is easy to see. Automorphisms of $V$ are just bijective linear maps $f: V \rightarrow V$, while automorphisms of $(V, g)$ are bijective linear maps $f: V \rightarrow V$ such that $g(x, y)=$ $g(f(x), f(y))$ for each $x, y \in V$.
} 
structure than Neo-Newtonian (or Galilean) spacetime. ${ }^{21}$ As one can readily appreciate, SYM* adequately captures our intuition concerning which mathematical space has more structure at least when applied to certain easy cases. Unfortunately, the case of metric structure and symplectic structure is not one of these easy cases of structural comparison. According to SYM*, these two types of structure are incomparable.

\subsubsection{Symplectic and Metric Structure are Incomparable}

Recall North's argument that metric structure is more structure than symplectic structure. The argument points out the relationships that the two structures bear to volume structure. A symplectic form on a manifold gives rise to volume structure. It naturally determines a volume form on the manifold. But volume structure, as North rightly points out, is less structure than metric structure. A metric gives rise to a notion of volume, but not vice versa. ${ }^{22}$ North concludes that symplectic structure is less structure than metric structure.

The problem with this argument is readily apparent: A symplectic form is not the same thing as a volume form. Symplectic structure is, in a strong sense, more structure than volume structure. If metrics and symplectic forms are both more structure than volume structure, then one cannot deduce from this alone that symplectic structure is less structure than metric structure. Metric structure and symplectic structure are, in fact, incomparable according to SYM*. Metric structure is not more structure than symplectic structure, but also symplectic structure is not more structure than metric structure.

This can be seen by considering the following two examples. For simplicity, we restrict attention to the case of inner products and symplectic forms on vector spaces rather than metrics and symplectic forms on manifolds. But of course, all of this generalizes easily to the case of manifolds. One is invited to think of the vector space $V$ as the tangent space $T_{p} \mathcal{M}$ to a manifold $\mathcal{M}$ at a point $p \in \mathcal{M}$.

\footnotetext{
${ }^{21}$ The reader can easily verify this by inspecting Chapter 2 of (Earman, 1989).

${ }^{22}$ Specifically, as pointed out in footnote 9 , a metric gives rise to a volume element on the manifold.
} 
Example 4 (Symplectic $\ngtr$ Metric). Consider a 2-dimensional real vector space $V$. Let $g: V \times V \rightarrow \mathbb{R}$ be an inner product on $V$, and $\Omega: V \times V \rightarrow \mathbb{R}$ be a symplectic form on $V$. Let $\left\{e_{1}, e_{2}\right\}$ be a arbitrary basis for $V$. Define $f_{1}: V \rightarrow V$ by

$$
\begin{aligned}
& f_{1}\left(e_{1}\right)=\frac{1}{2} e_{1} \\
& f_{1}\left(e_{2}\right)=2 e_{2},
\end{aligned}
$$

and extending linearly. One can easily verify that $f_{1}$ preserves the symplectic form $\Omega$ :

$$
\Omega(x, y)=\Omega\left(f_{1}(x), f_{1}(y)\right)
$$

for every $x, y \in V$. But $f_{1}$ does not preserve the inner product $g$. This can be seen simply by noting that $g\left(e_{2}, e_{2}\right) \neq 4 g\left(e_{2}, e_{2}\right)=g\left(f_{1}\left(e_{2}\right), f_{1}\left(e_{2}\right)\right)$.

Example 5 (Metric $\ngtr$ Symplectic). Let $V, g, \Omega$, and $\left\{e_{1}, e_{2}\right\}$ be as in Example 2. Define $f_{2}: V \rightarrow V$ by

$$
\begin{aligned}
& f_{2}\left(e_{1}\right)=e_{2} \\
& f_{2}\left(e_{2}\right)=e_{1},
\end{aligned}
$$

and extending linearly. One can easily verify that $g(x, y)=g\left(f_{2}(x), f_{2}(y)\right)$ for all $x, y \in V$. But $f_{2}$ does not preserve the symplectic form $\Omega$, as $\Omega\left(e_{1}, e_{2}\right) \neq-\Omega\left(e_{1}, e_{2}\right)=\Omega\left(f_{2}\left(e_{1}\right), f_{2}\left(e_{2}\right)\right)$.

To summarize, Example 4 presents an automorphism $f_{1}$ of the structure $(V, \Omega)$ which is not an automorphism of $(V, g)$. The map $f_{1}$ preserves the symplectic form, but 'breaks' the inner product structure. So we cannot apply $\mathrm{SYM}^{*}$ to conclude that a symplectic vector space has more structure than an inner product space. Example 5, on the other hand, presents an automorphism $f_{2}$ of the structure $(V, g)$ which is not an automorphism of $(V, \Omega) ; f_{2}$ preserves the inner product while 'breaking' the symplectic form. So we cannot apply SYM* to conclude that an inner product space has more structure than a symplectic vector space.

According the criterion SYM*, therefore, neither is a metric more structure than a symplectic form nor is a symplectic form more structure than a 
metric. The two structures are in this sense incomparable. At the very least, this discussion demonstrates that comparing metric structure and symplectic structure is not one of the easy cases of structural comparison. It is not like comparing the structure of a vector space to that of an inner product space, nor is it like any of the other easy cases enumerated above. Something more must be said in support of premise (P3). According to SYM*, (P3) is not true. ${ }^{23}$

\section{Two Methodological Remarks}

I hope to have demonstrated by this point that North's argument for (LS) is not sound. In general, Lagrangian mechanics does not presuppose nor give rise to a metric on configuration space. This means that in general, (P1) is not true. And even if (P1) were true, it would be hard to see a sense in which the Lagrangian statespace could have metric structure without the Hamiltonian statespace having the same. Furthermore, even if one conceded that both (P1) and (P2) were true, it is not at all clear that (LS) would then follow. Comparing metric structure and symplectic structure is not one of the easy cases of structural comparison. According to SYM*, (P3) is not true. In this final section I would like to make two brief methodological remarks which should be kept in mind by anyone trying to compare the structure that Lagrangian and Hamiltonian mechanics ascribe to the world.

\subsection{Statespace Realism}

In identifying the structure that Lagrangian mechanics and Hamiltonian mechanics impute to the world, one might be puzzled as to why we are looking at the structure of the statespaces of the theories. Recall the method that was used in the arguments for (P1) and (P2) above. We tried to show that

\footnotetext{
${ }^{23}$ If someone wants to argue that metric structure is more structure than symplectic structure, then the impetus is on them to provide a general criterion of structural comparison which gives this verdict. As North mentions (North, 2009, p. 87), DIM does give this verdict. According to DIM metric structure is more structure than symplectic structure. But as we have seen, DIM is not a general criterion, and it gives some unintuitive verdicts.
} 
Lagrangian mechanics imputed metric structure by showing that its statespace has metric structure. And likewise, we tried to show that Hamiltonian mechanics imputed symplectic structure by showing that its statespace has symplectic structure. One might balk, however, at the inference from "The statespace of theory $X$ has structure $Y$ " to "Theory $X$ ascribes structure $Y$ to the world."

Consider once again the case of spacetime theories. The sense in which spacetime theories ascribe structure to the world is fairly clear. For example, in absolute Newtonian spacetime there is a mathematical structure which allows one to identify spatial points over time. ${ }^{24}$ Neo-Newtonian spacetime does not have this structure. These two theories therefore tell us different things about the structure of spacetime.

The sense in which the mathematical structure of a theory's statespace ascribes structure to the world is not as clear as in the spacetime case. One might claim, I think reasonably, that a statespace in classical mechanics merely provides one with a convenient way of encoding the dynamical properties of bodies which are located in spacetime - the positions, velocities, and momenta of these bodies, for example. If this is the case, then it is hard to see how the mathematical structure of Lagrangian or Hamiltonian statespace imputes any structure whatsoever on the world. Certain statespace structures might make the description of what is happening in spacetime easier, but that does not mean that the world, in some sense, actually has the mathematical structure that these statespaces employ.

This serves to point out the extent to which this discussion of the structures that Lagrangian and Hamiltonian mechanics ascribe to the world relies on some form of statespace realism. ${ }^{25}$ If one thinks, as a statespace realist does, that statespace exists as a concrete thing,' then the mathematical structure of statespace ascribes structure to the world in exactly the same way that the structure of spacetime theories ascribes structure to the world. This is not the place for a critical evaluation of statespace realism. But it is important to mention that any argument seeking to demonstrate (LS) by

\footnotetext{
${ }^{24}$ Specifically, this structure is a unit timelike vector field which is covariantly constant (Earman, 1989, Ch. 2).

${ }^{25}$ North points this out as well (North, 2009, p. 80-81).
} 
appealing to the mathematical structure of the Lagrangian and Hamiltonian statespaces will rely on some form of statespace realism. ${ }^{26}$

\subsection{Physical Structure is not Mathematical Structure}

Recall that in North's argument for (LS) the following method was used to count the structure that a physical theory imputes: We take the physical theory, look at its models, and count the mathematical structure of the models. In general, using this sort of method to count the structure that a physical theory ascribes to the world requires great care. The following example illustrates a case where this method goes awry.

Example 6 (Special Relativity). Special relativity can be formulated in two equivalent ways: One can use a metric $g$ with signature $(+,-,-,-)$ or a metric $g^{\prime}$ with signature $(-,+,+,+) .{ }^{27}$ The only difference between these two formulations of special relativity is a sign convention. In the former, one defines timelike vectors $v$ as those vectors that satisfy $g(v, v)>0$; in the latter, one defines timelike vectors $v$ as those vectors that satisfy $g^{\prime}(v, v)<0$. One does not want to say that these two formulations of special relativity impute different structure on the world. But crucially, the models of these two theories are non-isomorphic. ${ }^{28}$

Example 6 has the following moral: In assessing the structure that a physical theory ascribes to the world, one cannot always just look to the mathematical structure of the models of the theory. ${ }^{29}$ This tactic would lead one to conclude that the two equivalent formulations of special relativity impute different, non-isomorphic structures to the world. ${ }^{30}$ And this would

\footnotetext{
${ }^{26}$ This is not to say, however, that any argument in support of (LS) must rely on statespace realism.

${ }^{27}$ Formally, it can formulated using a 4-dimensional $(1,3)$ metric affine space, or a 4 dimensional $(3,1)$ metric affine space. See (Malament, 2009) for the definition of a metric affine space.

${ }^{28}$ This is simply because two metric affine spaces of the same (finite) dimension are isomorphic if and only if they have the same signature (Malament, 2009, Prop. 2.3.4).

${ }^{29}$ Mathematical models for a physical theory might involve unnecessary mathematical structure. This is another (distinct) reason to be cautious when reading off physical structure from the mathematical structure of models.

${ }^{30}$ This is not to say that one who uses this tactic is committed to the claim that one formulation imputes more structure than the other.
} 
be an undesirable conclusion, to say the least. The difference between the two formulations is only a sign convention. That should not in any way affect the structure that the theory ascribes to the world.

The moral of Example 6 extends well beyond the case of Lagrangian and Hamiltonian mechanics. Anyone interested in assessing the structure that physical theories ascribe to the world should keep this in mind. One cannot read off the structure that a physical theory ascribes to the world simply from the mathematical structure of its models. One needs to remember the physical significance that certain bits of mathematics had for example, which vectors were defined as timelike vectors. It is naive to think that we can forget the physical significance that certain bits of mathematics employed by a physical theory have, and then still correctly identify the structure that the physical theory ascribes to the world. Such a method entails remarkably unintuitive conclusions, like the one in Example 6.

\section{Conclusion}

I hope to have shown that there are some significant obstacles that must be navigated by anyone interested in comparing the amount of structure that different physical theories ascribe to the world. The first type of obstacle arises in the task of identifying the kind of structure that each theory imputes, the second type in the task of comparing these kinds of structure. Unfortunately, in the case of Hamiltonian and Lagrangian mechanics, both types of obstacle have proven to be particularly troublesome. And I have argued here that neither has yet been successfully navigated.*

\section{References}

Abraham, Ralph, and Jerrold E. Marsden. (1978). Foundations of Mechanics. Reading, MA: Benjamin/Cummings Pub. Print.

Baez, John. (2005). "Lectures on Classical Mechanics." Manuscript.

\footnotetext{
${ }^{*}$ This material is based upon work supported by the National Science Foundation under Grant No. DGE 1148900.
} 
Earman, John. (1989). World Enough and Space-time: Absolute versus Relational Theories of Space and Time. Cambridge, MA: MIT. Print.

Mac Lane, Saunders. (1970). "Hamiltonian Mechanics and Geometry." The American Mathematical Monthly 77.6: 570-586.

Malament, David B. (2009). "Geometry and Spacetime." Web.

North, Jill. (2009). "The 'Structure' of Physics: A Case Study." Journal of Philosophy 106: 57-88.

Swanson, Noel, and Hans Halvorson. (2012). "On North's 'The Structure of Physics"". Manuscript.

Tao, Terence. (2008). "Phase Space." Web. 